\title{
Impact of psychological contract in knowledge sharing: A case study from Industrial Cluster Enterprises
}

\author{
Juanli Lan ${ }^{1}$, Bingxuan Wang ${ }^{2}$, Bingkun $\mathrm{Hu}^{3}$, Hongzhen $\mathrm{Lei}^{4}$ \\ 1-4 Shanxi Normal University, Xi'an, Shanxi, 710119, China
}

\begin{abstract}
Psychological contract plays a critical role in knowledge sharing. In this paper, we have analyzed the influence of psychological contract and affective commitment to knowledge sharing in China. After using AMOS software for data processing by employing data on the questionnaire, which indicated that there are high consistency and stability and good convergence validity and construction reliability among variables, the multiple Ordinary Least Square(OLS) method was utilized in the study. There are 183 questionnaires were received in the experiment. After analysis, 157 questionnaires were valid for the research; the effective recovery rate was about to $86 \%$. Based on the study, the conclusion could be conducted that the relationship contract and development contract of enterprise technical staff have positive impacts to knowledge sharing, On the contrary, the transaction contract has a negative impact to knowledge sharing.
\end{abstract}

\section{Introduction}

A large number of empirical studies show that the transfer of cluster knowledge is an important factor to improve the innovation ability and rapid development, which guarantees cluster enterprises to enhance their competitive advantage, and knowledge sharing is the key guarantee of knowledge transfer (Zhang Xiaobo, 2011; Cui Ning, 2014; Wang Bin, 2015; Wang Xin, 2016) ${ }^{[1-4]}$. With the increasingly important role of knowledge resources in enterprise competition, the research on knowledge sharing among enterprises in industrial clusters has become a hot issue in organizational planning and strategic development. Large number of studies show that the knowledge sharing among technicians in cluster enterprises has an increasing impact on the ability and quality of enterprise innovation. We chose the technical personnel of cluster enterprises to analyze the relationship among psychological contract, affective commitment and knowledge sharing.

\section{The theoretical basis and hypotheses}

\subsection{The influence of Psychological contract on knowledge sharing}

Reasonable use of psychological contract can have an imperceptible impact on employees in enterprises, and can obtain more positive and effective results than traditional incentive methods. Hendriks(1999) found that the knowledge sharing of employees is influenced by some psychological motives, such as sense of accomplishment, self-worth, responsibility and other factors ${ }^{[5]}$. Siemsen et al. (2007) found that only when the personal benefits gained from knowledge sharing were greater than the cost of knowledge sharing, employees would have a positive willingness to share knowledge ${ }^{[6]}$. Liang Qihua et al. (2006) pointed out that the unmeasurable of tacit knowledge lead to the negative effect of psychological contract violation on tacit knowledge transmission ${ }^{[7]}$. He Mingrui et al. (2011) under the conditions of China, took knowledge employees as the object, explored the difference of the influence of different dimensions of psychological contract on tacit knowledge sharing and also examined the applicability of the mature three-dimensional division of psychological contract abroad in China's national conditions ${ }^{[8]}$. Wang Shihong et al. (2012) focused on national auditors to explore the relationship between psychological contract and knowledge-sharing behavior. The results showed that auditors' psychological contract had a positive effect on their knowledge-sharing behavior $^{[9]}$. Lu Fuchai et al. (2012) proved that good trust relationship between enterprises and employees can have a further impact on psychological contract and knowledge sharing intention ${ }^{[10]}$.

H1-1:Transaction contract of technicians can positively affect explicit knowledge sharing

H1-2:Relationship contract of technicians can positively affect explicit knowledge sharing

H1-3:Development contract of technicians can positively affect explicit knowledge sharing

H1-4:Transaction contract of technicians can positively affect tacit knowledge sharing

H1-5:Relationship contract of technicians can positively affect tacit knowledge sharing 
H1-6:Development contract of technicians can positively affect tacit knowledge sharing

\subsection{The influence of Psychological contract on affective commitment}

Research is divided into the following aspects:(1).Psychological contract and affective commitment, Roussaeu et al. (1999) pointed out that the commitment of the members of the organization stems from their psychological contract. The degree of satisfaction of members' psychological contract can strengthen their affective commitment ${ }^{[11]}$. Turnley et al. (1999) suggested that when the psychological contract of the members of the organization is effectively maintained and the members feel that their expectations are satisfied, members will strengthen their commitment to the organization, etc. ${ }^{[12]}$. Wei Jiangru et al. (2011) pointed out through empirical study that scientific management can improve the psychological contract of employees, and then affect the level of employee commitment ${ }^{[13]}$. Xu Xixiong et al (2011) studied the relationship between psychological empowerment, support and commitment from the point of view of psychological contract, and demonstrated the application of psychological contract and commitment theory in practice through practical cases. It is proved that the reasonable construction of psychological contract has a positive effect on commitment ${ }^{[14]}$.(2). Psychological contract breach and affective commitment, Bunderson et al. (1993) pointed out that psychological contract breach will reduce the degree of commitment of members ${ }^{[15]}$. Qi Lin et al. (2012) pointed out that if members feel that the expected commitment has not been fulfilled, they will reduce their affective commitment to the enterprise, which will lead to a reduction in the level of work of their members and even the departure of members ${ }^{[16]}$.(3). The differences between psychological contract and affective commitment, and the differences between Chinese and western countries. Ling Wenshuan et al. (2000) pointed out that the unwritten relationship between employees and organizations is the common feature of psychological contract and affective commitment. The difference between the two is that the psychological contract is the implicit expectation between the employees and the organization, while the affective commitment is the willingness of the employee to contribute to the organization because of factors other than the economic contract ${ }^{[17]}$. Yu Shan et al. (2008) tested the difference between Chinese and western employees' feelings that psychological contract breach is the influence on affective commitment and work behavior by empirical study. The influence of different dimensions of psychological contract on Chinese and western employees is different ${ }^{[18]}$.

H2-1:Transaction contract of technicians have a positive impact on affective commitment

H2-2:Relationship contract of technicians have a positive impact on affective commitment

H2-3:Development contract of technicians have a positive impact on affective commitment

\subsection{The influence of affective commitment on knowledge sharing}

The research shows that affective commitment is binding on the members of the organization, and the level of affective commitment will affect the behavior of individuals (Meyer,2002) ${ }^{[19]}$. Low level of affective commitment may cause employees to deliberately make certain adverse behaviors ${ }^{[20]}$; high level of affective commitment has a positive role in promoting employee innovation, organizational citizenship behavior and so on ${ }^{[21]}$. According to the social exchange theory, they are willing to transmit knowledge to repay the enterprise ${ }^{[22]}$. When members agree with their own organization and have values consistent with the organization, they have a positive attitude towards the transmission of knowledge ${ }^{[23]}$. Low affective commitment can even hinder the knowledge-sharing process of employees. Wang Duanxu et al. (2009) focused on the IT industry and discussed affective commitment and knowledge sharing get positive correlation between two dimensions $^{[24]}$. Jin Hui (2013) studied the relationship between affective commitment and attitude and willingness to share knowledge ${ }^{[25]}$.

H3-1:Affective commitment of technicians have a positive impact on explicit knowledge sharing

H3-2:Affective commitment of technicians have a positive impact on tacit knowledge sharing

\subsection{The intermediary role of affective commitment}

This paper attempts to introduce the medium variable affective commitment to open the "black box" between psychological contract and knowledge sharing of technicians. Turnley (2003) pointed out that the employee's psychological contract contains the employee's expectation to the organization, the organization provides employees with a good environment to meet their expectation, will cause the employee to produce the high level emotion commitment, strengthens the employee's organizational citizenship behavior and knowledge sharing is also an important part of organizational citizenship behavior ${ }^{[26]}$. Zhang Aiwu (2006) constructed a psychological contract and knowledge sharing research model, and through the questionnaire analysis and other empirical research methods, briefly discussed the relationship between psychological contract, affective commitment, knowledge sharing [27]. We infer that the level of affective commitment of technicians leads to differences in the attitude of employees to work.

H4-1:The affective commitment of technicians plays an intermediary role in the relationship between transaction contract and explicit knowledge sharing

H4-2:The affective commitment of technicians plays an intermediary role in the relationship between relationship contract and explicit knowledge sharing

H4-3:The affective commitment of technicians plays an intermediary role in the relationship between development contract and explicit knowledge sharing 
H4-4:The affective commitment of technicians plays an intermediary role in the relationship between transaction contract and tacit knowledge sharing

H4-5:The affective commitment of technicians plays an intermediary role in the relationship between relationship contract and tacit knowledge sharing

H4-6:The affective commitment of technicians plays an intermediary role in the relationship between development contract and tacit knowledge sharing

\section{Research technique}

\subsection{Measurement of variables}

A total of 183 questionnaires were recovered, 157 valid questionnaire data were obtained, and the effective recovery rate was about $86 \%$. Correlation coefficents of main research variables show the results of these correlations are consistent with our theoretical expectations.

The measurement of psychological contract in this paper refers to the scale developed by Rousseau in $2000^{[28]}$. Psychological contract is divided into three parts in this paper:transaction contract, relation contract and development contract for 17 issues. In the study of Allen \& Meyer (1993) for the measurement of affective commitment ${ }^{[29]}$, Combining with the contents of the research, finally 6 questions. Knowledge sharing is divided into two parts: tacit knowledge sharing $(5$ questions) and explicit knowledge sharing(4 questions) respectively(Bock,2005) ${ }^{[30]}$.

\subsection{Regression Analysis and Mediation Test}

After using AMOS software for data processing, which indicated that there are high consistency and stability and good convergence validity and construction reliability among variables.

(1) Regression analysis on psychological contract to knowledge sharing among technicians

(a) Regression analysis on psychological contract to explicit knowledge sharing

The multiple linear regression analysis was performed with the substitution equation. Table 1 shows the multivariate correlation coefficient between the three explanatory variables and explicit knowledge sharing is 0.774 , the determining coefficient $R^{2}$ is 0.599 , the adjusted $R^{2}$ is 0.591 , and the error of standard estimation is 0.53365 , which means three explanatory variables can explain the $59.1 \%$ variation of explicit knowledge sharing. The $\mathrm{F}$ value is 76.030 , and the significance level is 0.000 , which has passed the significance test. The relationship contract $(\beta=0.365 、 \mathrm{p}=0.002<0.05)$, shows the H1-2 is accepted. Development contract $(\beta=0.379$, $\mathrm{p}=0.001)$, the H1-3 is accepted. The transaction contract $(\beta=0.029, p=0.778>0.5), p$ value failed to pass the significance test, $\mathrm{H} 1-1$ is rejected.
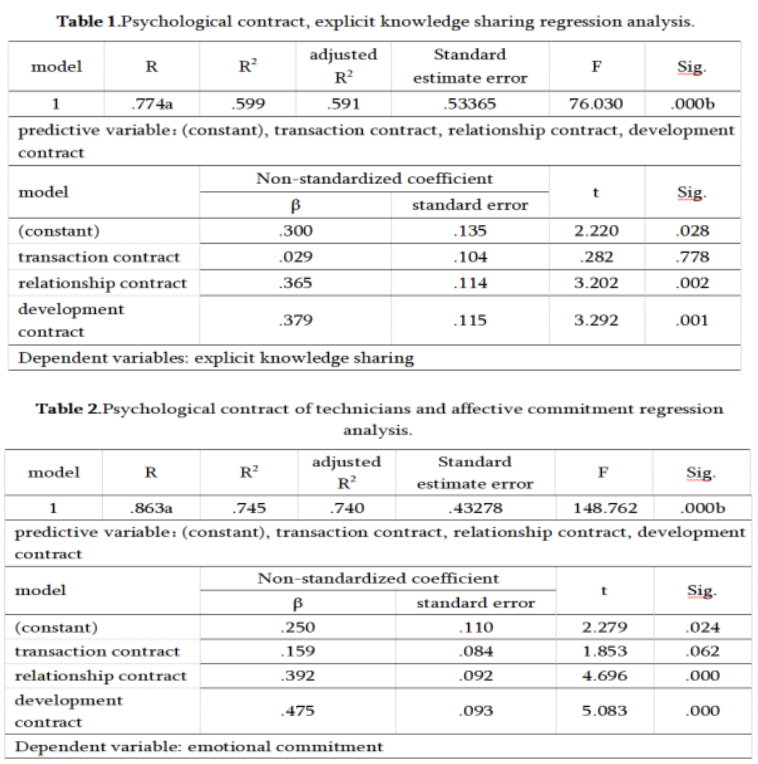

(b) Regression analysis on psychological contract to tacit knowledge sharing

Similarly, the multivariate correlation coefficient between the three explanatory variables and tacit knowledge sharing is 0.779 , the determining coefficient $\mathrm{R}^{2}$ is 0.606 , the adjusted $\mathrm{R}^{2}$ is 0.598 , and the error of the standard estimate is 0.49568 , which indicates that three explanatory variables can explain the $59.8 \%$ variation of tacit knowledge sharing. The $\mathrm{F}$ value of the equation is 78.481 and the significance level is 0.000 , which has passed the significance test. Among them, the transaction contract $(\beta=-0.237, p=0.027<0.5)$ shows that the H1-4 is rejected. The development contract $(\beta=0.391$, $\mathrm{p}=0.000<0.001)$ shows that the development contract can significantly affect tacit knowledge sharing, and the H1-6 is verified. The relationship contract $(\beta=0.108$, $p=0.266>0.5)$, the $p$ value failed to pass the significance test, H1-5 has not been validated.

(2) Regression analysis of psychological contract of technicians to affective commitment

Table 2 shows the multivariate correlation coefficient between the three explanatory variables and affective commitment is 0.863, the determining coefficient $\mathrm{R}^{2}$ is 0.745 , the adjusted $\mathrm{R}^{2}$ is 0.740 , and the standard estimate error is 0.43278 , assuming that three explanatory variables can explain $74.0 \%$ of the variation of affective commitment. The $\mathrm{F}$ value was 148.762 , and the significance level was 0.000 , which had passed the significance test. Among them, the transaction contract $(\beta=0.159, p=0.062>0.05)$ shows that the H2-1 is rejected. The relationship contract $(\beta=0.392, p=0.000$

$<0.01$ ) shows assuming $\mathrm{H} 2-2$ is verified. The development contract $(\beta=0.475, p=0.000<0.01)$, the $p$ value failed to pass the significance test, indicating that $\mathrm{H} 2-3$ is verified.

(3) Regression analysis of affective of technicians commitment to knowledge sharing

(a) Regression analysis of affective commitment of technicians to explicit knowledge sharing

Table 3 shows that the univariate correlation coefficient between explanatory variables and explicit knowledge sharing is 0.703 , the determining coefficient 
$\mathrm{R}^{2}$ is 0.494 , the adjusted $\mathrm{R}^{2}$ is 0.491 , and the error of standard estimation is 0.59520, assuming that explanatory variables can explain $49.1 \%$ variation of explicit knowledge sharing. The $\mathrm{F}$ value is 151.354 and the significance level is 0.000 . It can be concluded that the change of affective commitment as independent variable entering the regression equation reflects the linear change of dependent variable explicit knowledge sharing. The commitment $(\beta=0.691, \mathrm{p}=0.000<0.01)$, H31 is valid.

(b)Regression analysis of affective commitment of technicians to tacit knowledge sharing

Similarly, the test results show the univariate correlation coefficient between the explanatory variable and tacit knowledge sharing is 0.683 , the determining coefficient $\mathrm{R}^{2}$ is 0.466 , the adjusted $\mathrm{R}^{2}$ is 0.463 , and the error of standard estimation is 0.57324 . It is indicated that the explanatory variable can explain $46.3 \%$ variation of tacit knowledge sharing. The $\mathrm{F}$ value is 135.438 , and the significant level is 0.000 . The affective commitment ( $\beta=0.630, p=0.000<0.01), \mathrm{H} 3-2$ is accepted.

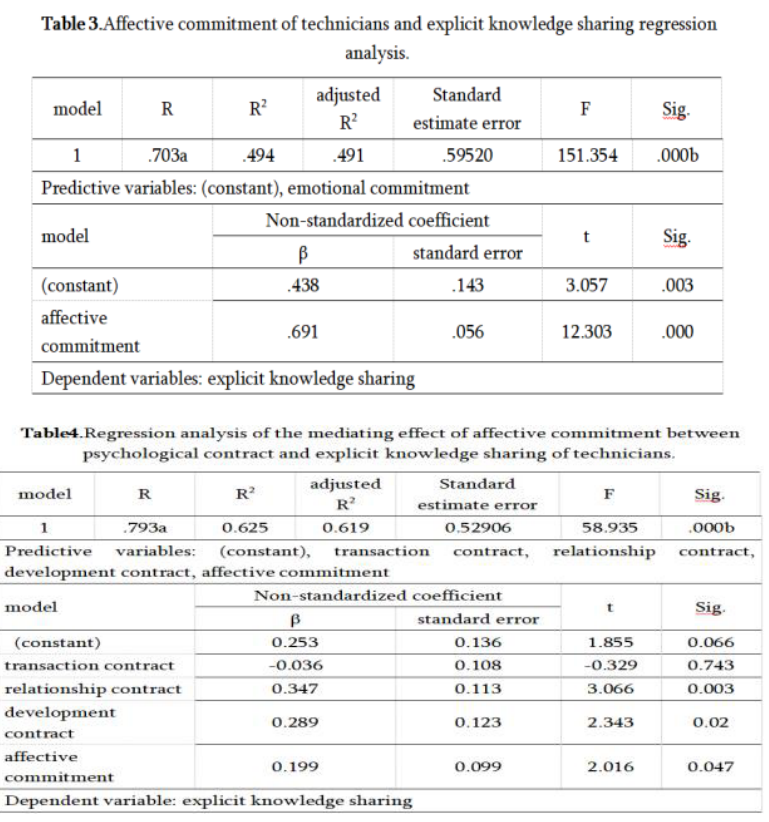

(4) The test of the intermediary function of affective commitment

(a) Affective commitment on psychological contract and explicit knowledge sharing of technicians

Table 4 shows that affective commitment coefficient ( $\beta=0.199)$, significant level $(\mathrm{Sig} .=0.047)$ is less than 0.05 . Because the relationship between transaction contract and explicit knowledge sharing is not significant in the previous analysis (Sig. $=0.778>0.05), \mathrm{H} 4-1$ is rejected. Coefficient of relation contract $(\beta=0.347)$, and reached the level of significance (Sig. $=0.003$ ). Therefore, affective commitment has partial intermediary utility between relationship contract of technicians and explicit knowledge sharing, and the H4-2 is accepted. The coefficient of the development contract $(\beta=0.289)$ and the significance level (Sig. $=0.02$ ), the H4-3 is accepted.

(b)Affective commitment on psychological contract and tacit knowledge sharing of technicians
Similarly, the results show that the coefficient of affective commitment $(\beta=0.201)$, and the level of significance (Sig. $=0.043<0.05$ ). Because the effect of transaction contract on tacit knowledge sharing is significant, but transaction contract on affective commitment is not, it is necessary to carry out Sobel test and the significance of the test result is 0.154 , more than $0.1, \mathrm{H} 4-4$ is rejected. Since the relationship contract has no significant influence on tacit knowledge sharing in the previous analysis (Sig. $=0.226>0.05), \mathrm{H} 4-5$ is rejected. The coefficient of development contract $(\beta=0.361)$, and reached the level of significance (Sig. $=0.002$ ), H4-6 is accepted.

\section{Conclusions and suggestions}

The main findings are as follows: (1) the relationship contract and development contract in the psychological contract of technical staff of an Enterprise can positively affect explicit knowledge sharing, but there is no significant influence between transaction contract and explicit knowledge sharing; (2)the development contract of technical personnel psychological contract has a significant positive predictive effect on tacit knowledge sharing, but the transaction contract can negatively predict tacit knowledge sharing, and there is no significant influence between relational contract and tacit knowledge sharing; (3) affective commitment plays an intermediary role in the relationship between technical personnel contract and explicit knowledge sharing, between development contract and explicit knowledge sharing, and between development contract and tacit knowledge sharing.

Therefore, in order to better promote the effect of knowledge sharing among technicians in cluster enterprises, and finally to improve the competitive advantage of industrial clusters. Firstly we should pay close attention to the psychological contract construction of enterprise technicians; Secondly, give the technicians enough affective commitment. Facing the fierce competition, complex and changeable realistic factors, enterprise managers should let the technicians realize their importance to the enterprise and enhance their sense of belonging; Lastly, set up the social network relation resources for the growth and development of the industrial cluster, improve the embedding of the cluster network, enlarge the network scale of the industrial cluster.

\section{Acknowledgment}

This research was financially supported by China Postdoctoral Science Foundation's 63rd Batch of Funding Projects (2018M633446).

\section{References}

1. Zhang Xiaobo,Ruan Jianqing.(2011)Evolution and Development of Industrial Clusters in China Zhejiang University Press,Zhejiang. 
2. Cui Ning,Shen Yuzhi,Zhang Haitao.(2014)Research on the Dynamic Mechanism Model of KnowledgeTransfer in Industrial Clusters.Science and Technology and Industry, 14(6): 78-80.

3. Wang Bin.(2015)Research on the Evolution of the Potential Difference of Knowledge Stock from the Perspective of Network Structure.Information Theory and Practice,38(1): 49-52.

4. Wang Xin,Liu Wei, Li.(2016)Research on Influencing Factors of Knowledge Transfer of Industry-University-Research Collaborative Innovation based on Dynamic Capability Theory .Information Science, 34(7): 36-40.

5. Hendriks P.(1999)Why Share Knowledge? The Influence of ICT on the Motivation for Knowledge Sharing. Knowledge \& Process Management,6(2):91-100.

6. Siemsen E,Balasubramanian S, Roth A V.(2007) Incentives that Induce Task-Related Effort, Helping, and Knowledge Sharing in Workgroups.Management Science, 53(10):15331550 .

7. Leung Kai-wah, Yu Kwong-shing.(2006)Enterprise Tacit Knowledge Transformation and Sharing Management Based on Psychological Contract. Research and Development Management, 18(1):7785.

8. Ho Ming-Rui,Lee Wing-kin.(2011)An Empirical Study on the Influence of Psychological Contract Types on Tacit Knowledge Sharing Willingness. Journal of Management, 8(1):56.

9. Lu Fuchai,Chen Xiaofeng.(2012)Knowledge Employees' Psychological Contract,Organizational Trust and Knowledge Sharing Intention. Economy Managment,(4):76-83.

10. Becerra-Fernandez I., Sabherwal R.(2014)Knowledge Management:Systems and Processes. Routledge.

11. Lingwen,Zhang Zhiyu,Fangziluo.(2000)A Study on the Structural Model of Organizational Commitment of Employees in China.Journal of Management Science, 3(2):76-81.

12. Rousseau D M, Tijoriwala S A.(1999)What's a Good Reason to Change? Motivated Reasoning and Social Accounts in Promoting Organizational Change.Journal of Applied Psychology,84(84):514528.

13. Turnley W H, Feldman D C.(1999)A Discrepancy Model of Psychological Contract Violations. Human Resource Management Review, 9(3):367386.

14. Wei Jiangru, Zhu Guoyun, Liu Ning.(2011)Research on the Influence of Knowledge Worker's Psychological Contract on Organizational Citizenship Behavior.Information Magazine, 30(2):115-118.

15. $\mathrm{Xu}$ Xixiong,Gan Weiyu.(2011)The Fit of Organizational Support,Psychological Empo werment and Employee Organizational Commitment: A Theoretical Framework of Employment Relationship Management for New Generation Peasant Workers-A Case Study Based on HaiDiLao. Management World, (12):131-147.

16. Bunderson J S.(2001)How Work Ideologies Shape the Psychological Contracts of Professional Employees: Doctors' Responses to Perceived Breach. Journal of Organizational Behavior, 22(7):717-741.

17. Qi Lin, Liu Zewen.(2012)The Influence of Psychological Contract Destruction on Employees' attitude and Behavior.Progress in Psychological Science,20(8):1296-1304.

18. Yu Shan, Chen Xiaohong.(2008)A Cross-cultural Comparison between China and the United States on the Psychological Contract of Employees and Their Attitudes to Work after Violating the Contract.System Engineering, 26(2):53-61.

19. Meyer J P, Stanley D J, Herscovitch L, et al.(2002) Affective, Continuance, and Normative Commitment to the Organization:A Meta-analysis of Antecedents, Correlates and Consequences. Journal of Vocational Behavior, 61(1):20-52.

20. Zhou Hao, long Lirong, Wang Yuqing.(2016)Overall Sense of Justice,Emotional Commitment and Employee Deviating Behavior: An Analysis Based on a Multi-object Perspective.Management Review,(11):162-169.

21. Liu Yuan, Zhou Zucheng.(2015)The Relationship between Employees' perceived Corporate Social Responsibility, Emotional Commitment and Organizational Citizenship Behavior-the Crosslayer Regulatory Role of Commitment-oriented Human Resource practice.Management Review,27(10):118-127.

22. Liu Xiaoping and Wang Zhongming.(2002)Organizational Commitment andIts Formation under the Background of Chinese and Western Cultures. Foreign Economy and Management, 24(1):17-21.

23. Lu L, Leung K, Koch P T.(2006)Managerial Knowledge Sharing: The Role of Individual, Interpersonal, and Organizational Factors.Management and Organization Review, 2(1):15-41.

24. Wang Duanxu,Zhu Xiaojing,Wang Ziwei.(2009)An Empirical Study on the Influence of Team Commitment on R\&D Personnel's Creativity:Knowledge Sharing as An Intermediary Variable. Science and Management of Science and Technology, 30(12):184-187.

25. Jin Hui, Yang Zhong, Huang Yanting, et al.(2013)The Mechanism of Organizational Motivation and Organizational Culture on Knowledge Sharing-based on the Revised Social Impact Theory. Scientific Research, 31(11):16971707. 
26. Turnley W H,Bolino M C,Lester S W,et al. (2003)The Impact of Psychological Contract Fulfillment on the Performance of In-Role and Organizational Citizenship Behaviors.Journal of Management:Official Journal of the Southern Management Association,29(2):187-206.

27. Zhang Aiwu, Li Xiyuan.(2006)Organizationemployee Employment Relationship and Knowledge Sharing.Aconomy Management, (8):61-68.

28. Rousseau D M.(2000)Psychological Contract Inventory:Technical Report.Carnegie Mellon University.

29. Allen N J, Meyer J P.(1993) Organizational Commitment: Evidence of Career Stage Effects?. Journal of Business Research, 26(1):49-61.

30. Bock $\mathrm{G}$ W,Zmud $\mathrm{R}$ W,Kim $\mathrm{Y}$ G,et al. (2005)Behavioral Intention Formation in Knowledge Sharing: Examining the Roles of Extrinsic Motivators,Social-Psychological Forces, and Organizational Climate.Mis Quarterly, 29(1):87-111. 